\title{
Intelligent Banknotes Recognition Based on Support Vector Machine (SVM)
}

\author{
Shafaf Ibrahim, Nur Zahidah Ibrahim \\ Faculty of Computer and Mathematical Sciences, Universiti Teknologi MARA, Jasin, Melaka, Malaysia \\ shafaf2429@uitm.edu.my, zahidahibrahim97@gmail.com
}

\begin{abstract}
Banknotes recognition is one type of intelligent system that is essential in today's modern world. Yet, it remains as a challenging task as the banknote may suffer from defects and the images are distorted during acquisition, resulting the need for a robust recognition system to mitigate these shortcomings. Additionally, more than 200 different currencies are being used in different countries around the world which might lead to confusion in differentiating these banknotes visually. Thus, this paper proposed a study of intelligent banknotes recognition based on Support Vector Machine (SVM). The main goal of this study is to extract the features of the different types of banknotes for efficient recognition. The color features were extracted using Histogram Equalization (HE) which are Red, Green and Blue (RGB) valuesin analysing the characteristics of five types of banknotes which are 100 Singapore Dollar, 100 Malaysia Ringgit, 100 Nepal Rupee, 100 Bangladesh Taka, and 100 China Yuan. The performance of the proposed study is evaluated to 150 testing images which produced $97.33 \%$ of overall mean recognition accuracy. It is believed that the study outcome could assist the financial institution particularly to recognize various types of banknotes conveniently and efficiently.
\end{abstract}

Key words: Banknotes, recognition, Histogram Equalization (HE), Support Vector Machine (SVM), image processing.

\section{INTRODUCTION}

A banknote is often referred to as a bill, paper money or just a note that is a kind of negotiable promissory note issued by a bank and payable to the bearer upon request. It can be used as medium of exchange of goods and services. The commercial banknotes were traded on the market served by the issuing bank only at face value [1]. A study in [2] claimed that monetary transaction is an integral part of our day to day activities. Societies ' strength in trading with other societies is highly dependent on the value that their currency holds.
Banknotes recognition is one type of intelligent system that is very important in today's modern world [3]. It is an image processing technology that is used to identify banknotes of various countries. Recognition of banknotes is an important pattern recognition area. Yet, it remains as a challenging task as the banknote may suffer from defects and the images are distorted during acquisition [4], resulting the need for a robust recognition system to mitigate these shortcomings.

There are a lot of banknotes throughout the world, in which each looks completely different. The size of the paper, for example, differs from the colour and pattern [5]. More than 200 different currencies are being used in different countries around the world which might lead to confusion in differentiating these banknotes visually. Besides, the manual banknotes recognition requires people to use their naked eyes to detect and recognize the different types of denomination of banknotes which may requires a lot of time [6]. The employees who work for financial institution need to distinguish the different banknotes types and this is not an easy task[7]. It is obligatory for them to remember each symbol of the banknotes which sometimes may lead to incorrect recognition [8], which induced the need of an efficient and accurate system to assist them [9].

Digital image processing is a method for performing certain image operations to enhance or to extract the essential information from it [10]. It is a form of signal processing in which input is an image and output may be image or image-related features. Image processing is used to pre-process and convert the image into a form suitable for further analysis [11]. It is increasingly used in many industries such as food processing, medical science, particle technology, engineering, and many more [10].

In another note, Support Vector Machine (SVM) is a supervised machine learning approach which is widely recognized for the small sample database as a statistical learning apotheosis [12]. The SVM has demonstrated its excellent ability to learn and generalize and has worked extensively in many areas. Support vectors are the vectors that define the hyperplane. It finds the hyperplane that maximizes the margin between the two classes can perform classification. 
Thus, based on the problems discussed, a study of banknotes image recognition based on SVM is proposed. The implementation of the proposed banknotes structure and features is believed to produce more proficient way to enhance the accuracy system for banknotes recognition in the future.

The organization of the rest of this paper is as follows: Section 2 presents our research method, including the description of banknotes images, as well as the proposed feature extraction and banknotes recognition techniques. Our results and discussions are presented in Section 3. Lastly, we present our findings in Section 4.

\section{RESEARCH METHODS}

This study aims to recognize the banknotes images based on SVM. Figure 1 depicts the flowchart of this research.

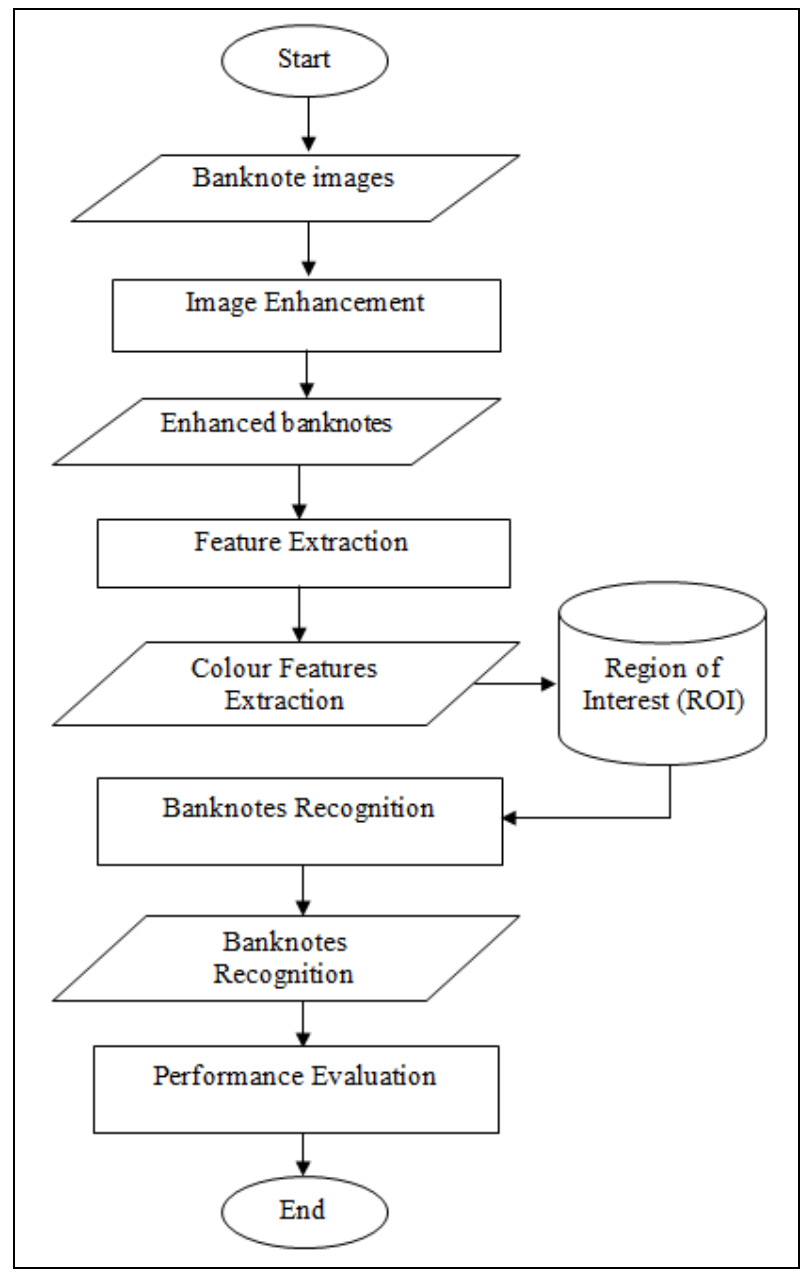

Figure 1:Flowchart of proposed research

The proposed flowchart of intelligent banknotes recognition begins with the input image of banknote. The image will then go through the image enhancement. Next, the processing consists of two sub-processes which are feature extraction and banknotes recognition. The feature extraction is used to extract the color feature properties of the banknote in the input image. Whereas, the recognition is used to recognize the type of banknote that the image belongs to. After the image is recognized, the system will produce the final result which is the banknote recognition before the recognition performance is evaluated.

\subsection{Banknote Images}

The banknote images were collected from the Kaggle dataset on the internet as recommended by [13]. The data types for the images are Portable Network Graphic (.PNG) or Joint Photographic Experts Group (.JPEG). A total of 150 banknote images in which 30 images for each banknote were collected. This study is only focusing on five types of country banknotes which are 100 Singapore Dollar, 100 Malaysia Ringgit, 100 Nepal Rupee, 100 Bangladesh Taka and 100 China Yuan. Table 1 tabulates the sample of banknote images used in this study.

Table 1:Samples of Banknote Images

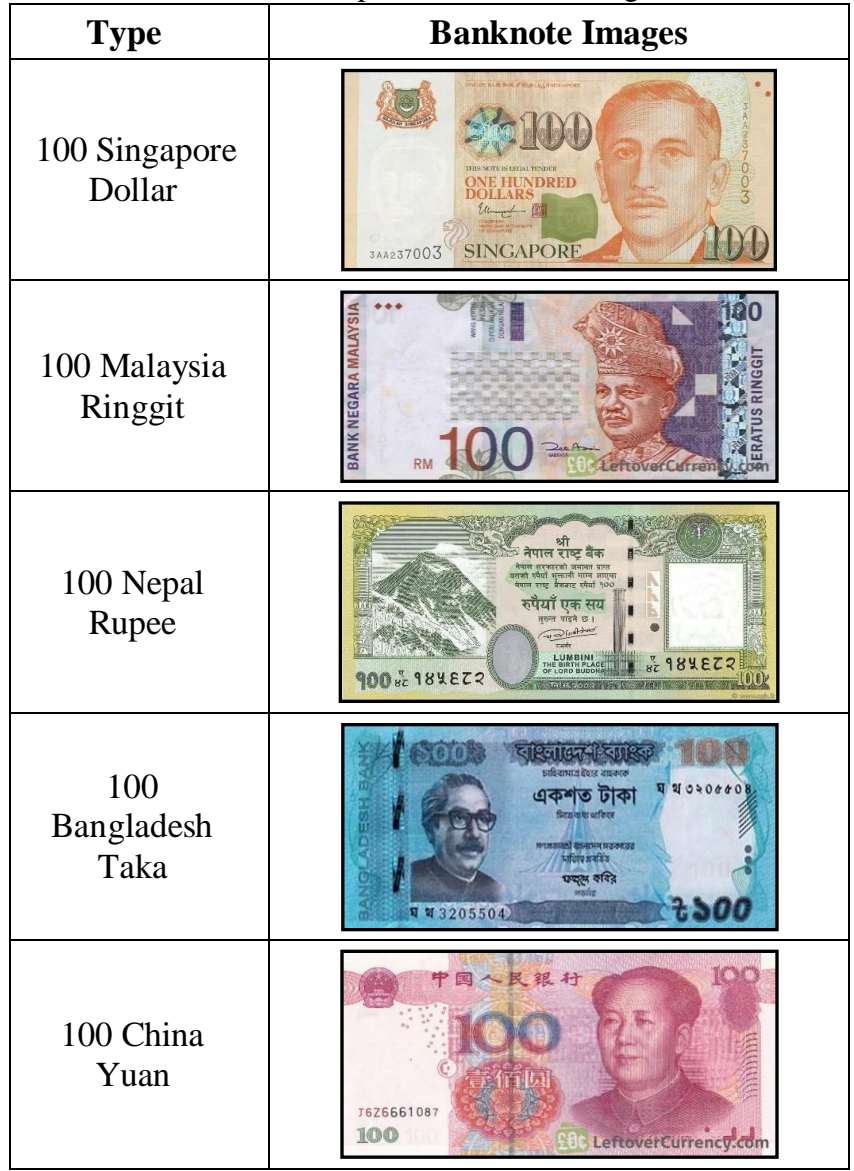

\subsection{Image Enhancement}

Image enhancement is a process for removing undesirable distortion due to unwanted noise, excessive intensity saturation and blurring effect due to degradation of contrast [14]. It produces better and better image quality [15]. The Histogram Equalization (HE) technique is implemented to reduce the noise and make it sharpen [16]. This is a point method that redistributes the intensity distributions of the 
banknote images in obtaining a uniform histogram for the banknotes image. It has been conducted in three steps which are histogram computation, normalized sum of histogram calculation, and input image to an output image transformation. Figure 2 depicts the image of banknotes image before and after the HE enhancement process.
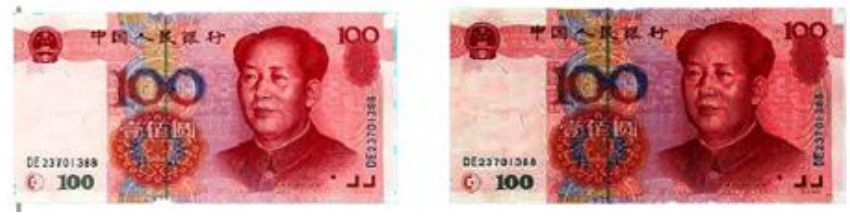

Figure 2:Banknotes before and after HE image enhancement

\subsection{Feature Extraction}

After the image has been enhanced, the next phase is feature extraction. The feature extraction is a vital element in image processing to extract the features by retaining as much information as possible from a large set of data [17]. Colour is one of the most important features of images that have been defined by a specific colour space or model. Colour Histogram is a widely used technique for an image retrieval feature [18]. It is very popular as it is trivial to calculate. Additionally, the small camera viewpoint changes do not affect the histogram [16]. In this study, the Colour Histogram is employed to extract the numbers of colour spaces which are Red, Green, and Blue (RGB). The RGB values are then be stored in the Region of Interest (ROI) table.

Table 2 tabulates the summary of ROI table which includes the range of the minimum and maximum value of RGB values obtained from the banknotes feature extraction. The ROI table is acted as a feeder in the SVM recognition subsequently.

Table 3: Summary of ROI Table

\begin{tabular}{|c|c|c|}
\hline Banknote & $\begin{array}{c}\text { Color } \\
\text { Features } \\
\end{array}$ & Range value \\
\hline \multirow{3}{*}{$\begin{array}{c}100 \\
\text { Singapore } \\
\text { Dollar }\end{array}$} & Red & $157.08-239.3$ \\
\hline & Green & $163.26-224.63$ \\
\hline & Blue & $130.78-199.49$ \\
\hline \multirow{3}{*}{$\begin{array}{c}100 \\
\text { Malaysia } \\
\text { Ringgit }\end{array}$} & Red & $154.34-211.45$ \\
\hline & Green & $133.92-199.18$ \\
\hline & Blue & $155.41-222$ \\
\hline \multirow{3}{*}{$\begin{array}{l}100 \text { Nepal } \\
\text { Rupee }\end{array}$} & Red & $149.96-197.9$ \\
\hline & Green & $155.2-202.97$ \\
\hline & Blue & $145.22-189.85$ \\
\hline \multirow{3}{*}{$\begin{array}{c}100 \\
\text { Bangladesh } \\
\text { Taka } \\
\end{array}$} & Red & $102.94-170.82$ \\
\hline & Green & $148.37-197.37$ \\
\hline & Blue & $157.15-215.52$ \\
\hline \multirow{3}{*}{$\begin{array}{c}100 \text { China } \\
\text { Yuan }\end{array}$} & Red & $175.77-240.92$ \\
\hline & Green & $131.35-200.54$ \\
\hline & Blue & $125.9-201.62$ \\
\hline
\end{tabular}

\subsection{Banknotes Recognition}

Recognition is a fundamental task to recognize or classify an image to their category [19]. The SVM is typically capable of delivering higher performance in terms of recognition accuracy as compared to the other recognition algorithms [20]. It classifies between two groups by constructing a hyperplane in a high-dimensional space that can be used for recognition. The banknotes recognition is conducted based on the technique of SVM. It works by mapping data into a high-dimensional feature space such that data points can be classified, even if the data could not be separated linearly. The hyperplane can be described in Eq. (1).

$w \cdot x i+b=0$

where:

$\mathrm{w}=$ normal a vector of the hyperplane

$\mathrm{b}=$ position of the relative area to the coordinate center

However, the dispersion of data that tends differently remains as a barrier in the recognition process, so linear separation will be difficult. Thus, SVM introduces the kernel function shows in Eq. (2) which converts the original data space into a new, higher dimensional space.

$K\left(x_{n}, x_{i}\right)$

In this study, the kernel used is Radial Basis Function (RBF). In some input space, the RBF kernel on two samples $x$ and $x^{\prime}$ is defined as feature vectors as defined Eq. (3).

$K\left(x, x^{\prime}\right)=\exp \frac{\|x-x\|^{2}}{2 \sigma^{2}}$

where:

$\left\|x-x^{\prime}\right\|^{2}=$ squared Euclidean distance between the two feature vector

$\sigma=$ free parameter

\subsection{Performance Evaluation}

The performance of the banknote recognition is evaluated using a truth table. It is carried out by comparing the banknote recognition result with the actual type of banknote. Based on the truth table obtained, the recognition accuracy for each type of banknote is calculated using Eq. (4):

$\%$ of Accuracy $=\frac{\text { No.of TRUE recognition result }}{\text { Total no.of testing images }} \times 100 \%$

\section{RESULTS AND DISCUSSIONS}

Thirty testing images are tested for each type of banknote. The performance of the SVM banknote recognition accuracy result is demonstrated in Table 4. 
Table 4:SVM Banknote Recognition Accuracy Result

\begin{tabular}{|c|c|c|c|}
\hline Banknote & $\begin{array}{c}\text { No. of } \\
\text { Testing } \\
\text { Image }\end{array}$ & $\begin{array}{c}\text { No. of } \\
\text { TRUE } \\
\text { recognition }\end{array}$ & $\begin{array}{c}\text { \% of } \\
\text { Accuracy }\end{array}$ \\
\hline $\begin{array}{c}\text { Singapore } \\
\text { Dollar }\end{array}$ & 30 & 30 & 100 \\
\hline $\begin{array}{c}\text { 100 } \\
\text { Malaysia } \\
\text { Ringgit }\end{array}$ & 30 & 30 & 100 \\
\hline $\begin{array}{c}\text { 100 Nepal } \\
\text { Rupee }\end{array}$ & 30 & 28 & 93.33 \\
\hline $\begin{array}{c}\text { 100 } \\
\text { Bangladesh } \\
\text { Taka }\end{array}$ & 30 & 29 & 96.67 \\
\hline $\begin{array}{c}\text { 100 China } \\
\text { Yuan }\end{array}$ & 30 & 29 & 96.67 \\
\hline MEAN & & $\mathbf{9 7 . 3 3}$ \\
\hline
\end{tabular}

Based on the Table 4, both 100 Malaysia Ringgit and 100 Singapore Dollar are monitored to produce excellent recognition accuracy at $100 \%$. It is followed by the 100 Bangladesh Taka and 100 China Yuan which also produced similar recognition accuracy at $96.67 \%$. The 100 Nepal Rupee is observed to return the lowest recognition accuracy at $93.33 \%$. It might be caused by the misrecognition in which the 100 Rupee Nepal is recognized as 100 Singapore Dollar, and 100 Taka Bangladesh has been misrecognized as 100 Rupee Nepal. There are also some cases where the 100 China Yuan is misrecognized as 100 Singapore Dollar. These occurred due to the slightly similar range values of extracted color features among each banknote. As a conclusion, the SVM based banknotes recognition is found to be effective as the accuracy for all banknotes obtained are all above $90 \%$. The overall mean percentage of accuracy is observed to produce an almost excellent percentage of accuracy which is $97.33 \%$.

\section{CONCLUSION}

This paper presents a study on banknotes recognition based on SVM. The color features of each banknote were extracted using the Colour Histogram in analyzing the color characteristics of the banknotes. On the different note, the SVM is used for the recognition of five types of banknotes which are 100 Singapore Dollar, 100 Malaysia Ringgit, 100 Nepal Rupee, 100 Bangladesh Taka, and 100 China Yuan. The application has been successful on a variety of test images. The performance of the banknotes recognition is evaluated using a truth table. The performance of the SVM recognition is tested to forty testing banknote images. The overall mean accuracy percentage demonstrated a very sharp accuracy at $97.33 \%$. It can be concluded that the proposed banknotes recognition based on SVM is found to be successful. However, it is recommended that more testing images and additional type of banknote images could be consider in the future.

\section{ACKNOWLEDGEMENT}

The research was supported by Ministry of Education Malaysia (MoE), and Universiti Teknologi MARA through the Fundamental Research Grant Scheme (FRGS) (600-IRMI/FRGS 5/3 (215/2019)).

\section{REFERENCES}

1. D. Mieczyslaw.The Global Currency Area a Way to Constructively End the Era of Reserve Currency, Modern Economy, Vol. 05, pp. 289-302, 2014.

2. K. Sawant and C. More. Currency Recognition Using Image Processing and Minimum Distance Classifier Technique, International Journal of Advanced Engineering Research and Science, vol. 3, no. 9, pp. 1-8, 2016.

3. S. Muhammad.An Intelligent Paper Currency Recognition System, Procedia Computer Science, Vol. 65, pp. 538-545, 2015.

4. T. Dittimi, A. Hmood, and C. Suen. Mobile based assistive technologies for authentication of banknotes, in ICPRAI 2018 International Conference on Pattern Recognition and Artificial Intelligence, Canada, 2018, pp. 366-371.

5. M. R. S. Lande, R. Malokar, H. Lajurkar, A. Karmore, and M. R. S. Lande, Currency Recognition Blind Walking Stick, Asian Journal of Convergence in Technology, vol. IV, no. I, pp. 4-7, 2018.

6. A. Arora, F. A. Kunroo, K. Shubham, R. Kumar, and D. R. R. Babu. Currency Recognition System Using Canny Edge Detection, Colour And Texture Analysis, J. Technological Advances and Scientific Res, vol. 2, no. 04, pp. 178-180, 2016.

7. S. D. Bharkad and A. A. Shirsath. Survey of Currency Recognition System Using Image Processing, International Journal of Computational Engineering Research, vol. 03, no. 7, pp. 36-40, 2013.

8. T. D. Pham, D. T. Nguyen, C. Park, and K. R. Park.Deep Learning-Based Multinational Banknote Type and Fitness Classification with the Combined Images by Visible-Light Reflection and Infrared-Light Transmission Image Sensors, Sensors, vol. 19, no. 4, pp. 792, 2019.

9. S. A. Bhavani.Currency Recognition using SIFT, International Journal of Computer Applications, vol. 167, no. 9, pp. 15-20, 2017.

10. S. Kumari, B. Prasad, and A. Nasim. Digital Image Processing in X-Ray Imaging, International Journal of Research in Engineering, Technology and Science, vol. VII, pp. 1-7, 2017.

11. H. N., Nguyen. A novel method for asphalt pavement crack classification based on image processing and machine learning, Engineering with Computers, vol. 35, pp.487-498, 2019. 
12. A. T. Azar and S. A. El-Said. Performance analysis of support vector machines classifiers in breast cancer mammography recognition, Neural Computing and Applications, vol. 24, no. 5, pp. 1163-1177, 2014.

13. A. Preez and J. Bekker. A Machine Learning Decision Support Framework for Industrial Engineering Purposes, in 2020 International Conference on Industrial Engineering and Industrial Management (IEIM 2020), Association for Computing Machinery, Paris, 2020, pp. 9-14.

14. V. Singh and A. K. Misra. Detection of plant leaf diseases using image segmentation and soft computing techniques, Information Processing in Agriculture, vol. 4, no. 1, 2017, pp. 41-49.

https://doi.org/10.1016/j.inpa.2016.10.005

15. T. L. S. Gowri and and T. Amudha. A Review on Mammogram Image Enhancement Techniques for Breast Cancer Detection, in 2014 International Conference on Intelligent Computing Applications, United States, 2014, pp. 47-51.

16. R. Kandwal, A. Kumar, and S. Bhargava. Existing image segmentation techniques, International Journal of Advanced Research in Computer Science and Software Engineering, vol. 4, no. 4, 2014.

17. K. Gaurav and P. K. Bhatia. A Detailed Review of Feature Extraction in Image Processing Systems, in 2014 Fourth International Conference on Advanced Computing \& Communication Technologies, India, 2014, pp. 5-12.

18. K. Mayathevar, M. Veluchamy, and B. Subramani. Fuzzy color histogram equalization with weighted distribution for image enhancement, Optik, vol. 216, pp. 164927, 2020.

19. Z. Chen, X. Wei, P.Wang, and Y. Guo.Multi-Label Image Recognition with Graph Convolutional Networks, IEEE Conference on Computer Vision and Pattern Recognition (CVPR),pp. 5177-5186, 2019.

20. Y. Wang, J. Chen, W. Tang, D. Xia, Y. Liang, and X. Li.Modeling adsorption of organic pollutants onto single-walled carbon nanotubes with theoretical molecular descriptors using MLR and SVM algorithms, Chemosphere, vol. 214, pp. 79-84, 2019. 